\title{
Machine Learning approach to the phase problem in Bragg Coherent Diffraction Imaging
}

\author{
Longlong $\mathrm{Wu}^{1,2}$, Shinjae $\mathrm{Yoo}^{1}$, Ana F. Suzana ${ }^{2}$, Tadesse A. Assefa ${ }^{2,3}$, Jiecheng Diao4, Ross J. Harder ${ }^{5}$, \\ Wonsuk Cha ${ }^{5}$ and lan K. Robinson ${ }^{2,4}$
}

\author{
${ }^{1}$ Computational Science Initiative, Brookhaven National Laboratory, Upton, NY 11973, USA \\ ${ }^{2}$ Condensed Matter Physics and Materials Science Department, Brookhaven National Laboratory, Upton, NY 11973, USA \\ ${ }^{3}$ Stanford Institute for Materials and Energy Sciences, SLAC National Accelerator Laboratory, Menlo Park, California 94025, USA \\ ${ }^{4}$ London Centre for Nanotechnology, University College London, London, WC1E 6BT, United Kingdom. \\ ${ }^{5}$ Advanced Photon Source, Argonne, Illinois 60439, USA
}

i.robinson@ucl.ac.uk

A solution to the crystallographic "phase problem" was proposed by David Sayre immediately after the announcement of the Shannon Information Theorem, requiring the diffraction to be sampled more than twice as finely as the Bragg peak spacing [1]. The implicit need for X-ray coherence has been happily solved with the development of the latest synchrotron sources, where Bragg Coherent Diffraction Imaging (BCDI) experiments are routinely performed. The fringed diffraction patterns can be oversampled so as to overdetermine the phase problem and iterative algorithms can converge on the solution. Despite meeting all the oversampling requirements of Sayre and Shannon, current projection-based iterative phase retrieval approaches still have trouble achieving a unique inversion of experimental data in the presence of noise. We propose to overcome this limitation by employing Machine Learning in a Convolutional Neural Network model which combines supervised training with unsupervised refinement. Remarkably, our model can be used without any prior training to learn the missing phases of an image based on minimization of an appropriate "loss function" alone. We demonstrate significantly improved performance with experimental Bragg CDI data over traditional iterative phase retrieval algorithms [1,2]. An example is shown in Figure 1, which compares the reconstructions of a cube-shaped $\mathrm{SrTiO}_{3}$ nanocrystal using the traditional iterative procedure with our new Machine learning approach.

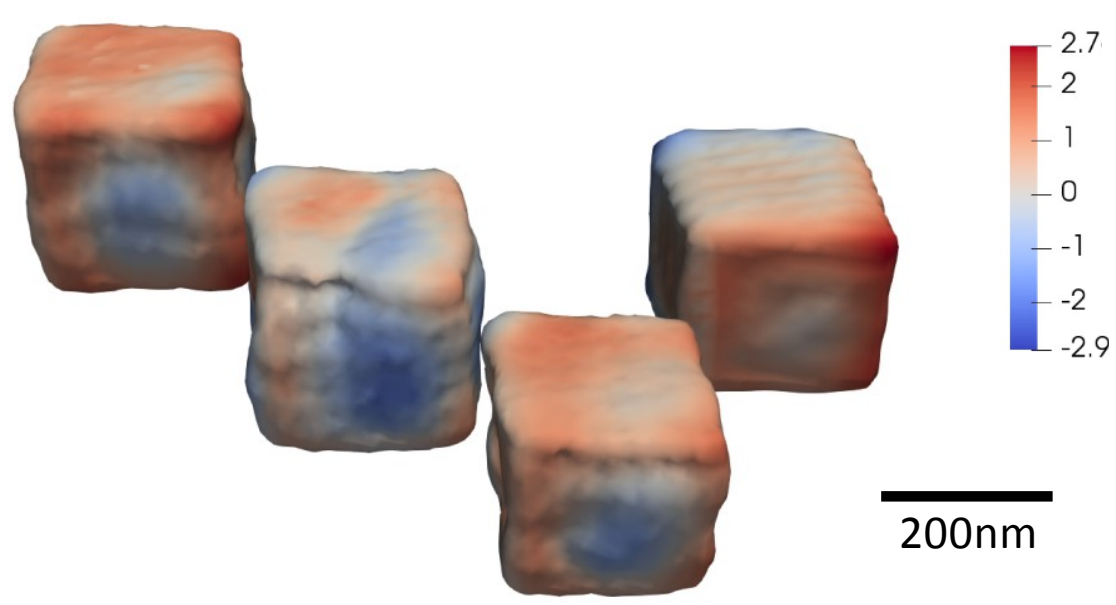

Figure 1. BCDI images of a $\mathrm{SrTiO}_{3}$ nanocrystal reconstructed with traditional "shrink wrap" interative algorithms (left three examples) and the new Machine Learning approach (right). Colors encode the image phase value on the surfaces in radians.

[1] Some implications of a theorem due to Shannon, D. Sayre, Acta Cryst. 5, 843 (1952).

[2] Complex Imaging of Phase Domains by Deep Neural Network Longlong Wu, Pavol Juhas, Shinjae Yoo and Ian Robinson, IUCrJ 8 12-21 (2021)

[3] 3D Coherent X-ray Imaging via Deep Convolutional Neural Networks, Longlong Wu, Shinjae Yoo, Ana F. Suzana, Tadesse A. Assefa, Jiecheng Diao, Ross J. Harder, WonsukCha and Ian K. Robinson,to be published

Keywords: Machine Learning; Phase problem; Nanocrystal Structure; Coherent Imaging 